\title{
Scanning Removal of Ion-implanted Novolak Resist by using a Laser Irradiation
}

\author{
Tomosumi Kamimura ${ }^{1}$, Yuta Kuroki ${ }^{1}$, Takuya Kiriyama ${ }^{1}$, Hiroki Muraoka ${ }^{1}$, \\ Takashi Nishiyama ${ }^{2}$, Yoshiyuki Harada ${ }^{1}$, Hiroyuki Kuramae ${ }^{1}$ and Hideo Horibe ${ }^{2}$ \\ ${ }^{1}$ Department of Electronics, Information and Communication Engineering, \\ Osaka Institute of Technology, 5-16-1 Ohmiya, Asahi-ku, Osaka 535-8585, Japan \\ ${ }^{2}$ Department of Applied Chemistry and Bioengineering, Graduate School of Engineering, Osaka City \\ University, 3-3-138 Sugimoto, Sumiyoshi, Osaka 558-8585, Japan
}

\begin{abstract}
Laser resist stripping for the ion-implanted novolak resist was successfully performed without occurring laser damage to the Si wafer. In order for the advanced laser resist stripping method to be successful, it is important for the pulsed laser beam to pass though the ion-implanted resist and absorb into the Si wafer surface. The novolak resists which are implanted with B, P, and As ions, respectively, were irradiated with a pulsed $532 \mathrm{~nm}$ laser. Regardless of the implanted ion species and density, more than $74 \%$ of the laser power was found to absorb into the Si wafer surface. For the laser irradiation of 1 pulse, the ion-implanted resist with a density of $5.0 \times 10^{13}$ atoms $/ \mathrm{cm}^{2}$ was completely stripped in the same way as that of a non-implanted resist. The optical absorption of the resist surface increased as the density of the ion-implantation increased. In case of the ion-implanted resist with a density of $5.0 \times 10^{15}$ atoms $/ \mathrm{cm}^{2}$, the resist was stripped by 20 pulses irradiation without occurring laser-induced surface damage. A scanning removal of the highly ion-implanted resist was also successfully stripped by using an optimized irradiation condition. A highly ion-implanted resist was continuously stripped by the scanning laser irradiation with 20 pulses.
\end{abstract}

Keyword: resist stripping, ion-implanted novolak resist, laser irradiation, laser-induced damage, scanning removal

\section{Introduction}

In semiconductor manufacturing, there is an ion implantation process in which 13 and 15 group elements are implanted to a substrate. In this process, the resist plays the role of a mask, and ions are implanted to the resist also. It is difficult to remove the ion-implanted resist because the resist is hardened, and is denatured by the implanted ions [1-3].

Resist removal from substrates in a semiconductor manufacturing process conventionally makes use of oxygen plasma and/or sulfuric acid hydrogen peroxide mixture (SPM). These environmentally unfriendly chemicals are used in large amounts and so cause environmental damage $[4,5]$.

In order to avoid using such chemicals, ozone with high oxidation potential is expected to remove the resist and organic residue. However, the resist cannot be removed using ozone alone at room temperature. The dry process using ozone requires temperatures higher than 250 in order to generate oxygen radicals from the ozone though thermal decomposition. For this reason, the process using ozone may oxidize substrates and metal wiring $[6,7,8]$.

The removal of the resist by laser 
irradiation instead of chemicals has the advantage of reducing environmental risks [9, 10]. An advanced laser resist stripping method for the positive-tone diazonaphthoquinone (DNQ) / novolak resist was successful developed without occurring laser damage to the Si wafer [11]. The pulsed laser irradiation in the water can improve the resist stripping effect when compared with that of conventional atmosphere irradiation. A laser irradiation of $532 \mathrm{~nm}$, having large photon energy was found to have higher resist stripping efficiency than that of the wavelength $1064 \mathrm{~nm}$. To apply this method to commercial contexts, ion-implanted photoresist is also surely removed from the $\mathrm{Si}$ wafer.

In this study, we have investigated the ion-implanted novolak resist removal by using laser irradiation. In order for the advanced laser resist stripping method to be successful, it is important for the pulsed laser beam to pass through the ion-implanted resist and absorb into the $\mathrm{Si}$ wafer surface. The optical characteristics of the ion-implanted resist were evaluated at the wavelength of $532 \mathrm{~nm}$. For this purpose, novolak resists which were implanted with $\mathrm{B}, \mathrm{P}$, and $\mathrm{As}$ ions with density from $5.0 \times 10^{13}$ atoms $/ \mathrm{cm}^{2}$ to $5.0 \times 10^{15}$ atoms $/ \mathrm{cm}^{2}$, respectively, were prepared in this experiment. A stripping condition in which damage did not occur to the $\mathrm{Si}$ wafer was confirmed in the highly ion-implanted sample. In addition, demonstration of the ion-implanted resist stripping with laser beam scanning was performed by using optimized conditions.

\section{Experimental Procedure}

Ion-implanted novolak resists used in this experiment were prepared using the following procedure. HMDS was spin-coated onto the Si wafer substrate using a spin-coater (ACT-300A from ACTIVE) at $3000 \mathrm{rpm}$ for $60 \mathrm{sec}$. Then, the positive DNQ / novolak resist (OFPR- 800 from Tokyo Ohka Kogyo Co., Ltd.) was spin-coated at $2000 \mathrm{rpm}$ for 20 sec. The resist was pre-baked at 100 degrees Celsius for $60 \mathrm{sec}$. The resist thickness was found to be from 1.0 to $1.1 \mu \mathrm{m}$ by profilometry (Dektak6M from ULVAC). B, P, and As ions, were implanted to the novolak resist surface with the acceleration energy of $70 \mathrm{keV}$. The density of the ion-implants was from $5.0 \times 10^{13}$ atoms $/ \mathrm{cm}^{2}$ to $5.0 \times 10^{15}$ atoms $/ \mathrm{cm}^{2}$.

Optical characteristic of ion-implanted resist was evaluated by using a second harmonics $(532 \mathrm{~nm})$ of a pulsed Nd:YAG $\left(\mathrm{Y}_{3} \mathrm{Al}_{5} \mathrm{O}_{12}\right)$ laser. The laser beam was irradiated to the $\mathrm{Si}$ wafer coated with ion-implanted resist. The relations between the irradiation power and the reflection power from the $\mathrm{Si}$ wafer coated with an ion-implanted resist were measured precisely. To calculate the absorption of ion-implanted resists, the same measurement was performed on the Si wafer surface only.

Prepared ion-implanted resists were sunk underwater to obtain an improved stripping condition identical to a non-implanted novolak resist. The pulsed laser was irradiated using a "1-on-1" method (irradiated place was changed every pulse). A lens with a focal length $\mathrm{f}=300 \mathrm{~mm}$ was used to focus the laser radiation onto the resist surface. The intensity of the pulses was varied with an attenuator consisting of a polarizer and a half-wave plate. The prepared ion-implanted resist was installed perpendicularly in a container having an aperture for laser beam incidence as shown in Figure 1. The distance from the water surface to the resist was controlled using the quantity of the water put in the container. The resist was irradiated with a laser beam of $532 \mathrm{~nm}$ in the water at a depth of $2 \mathrm{~mm}$.

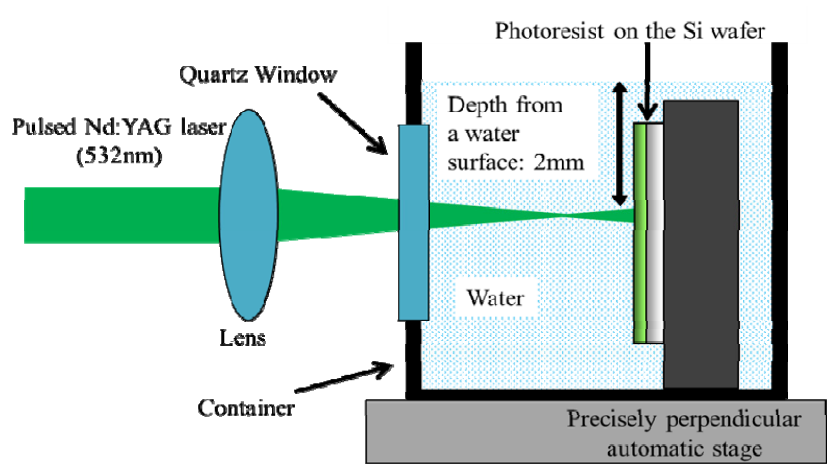

Figure 1. Experimental setup of the laser irradiation for ion-implanted resist stripping in the water.

\section{Results and Discussion}

The absorbance of the ion-implanted resist obtained from the optical characteristic 
measurement is summarized in Figure 2. At the wavelength of $532 \mathrm{~nm}$, novolak resist is transparent optically. The irradiated laser beam arrives at the interface of the $\mathrm{Si}$ wafer and the resist. For the non-implanted photoresist, approximately $90 \%$ of irradiated laser power was absorbed at the Si-resist interface. In contrast, the absorbance of ion-implanted resists was from 74 to $90 \%$. Even a highly ion-implanted resist with a density of $5.0 \times 10^{15}$ atoms $/ \mathrm{cm}^{2}$ showed the same absorbance of approximately $90 \%$ as the non-implanted resist. Ion-implanted novolak resists were also transparent optically in the wavelength of $532 \mathrm{~nm}$. No dependence on ion-implanted density and implanted ions species was identified in the difference of the absorbance between the samples. The single-layered novolak resist film having low index of refraction against a $\mathrm{Si}$ wafer works as AR coating. Thus, the difference of slight film thickness among the samples was thought to cause dispersion of the absorbance. From these results, irradiated laser power pass through the ion-implanted resist surface which changed in quality chemically and mechanically.

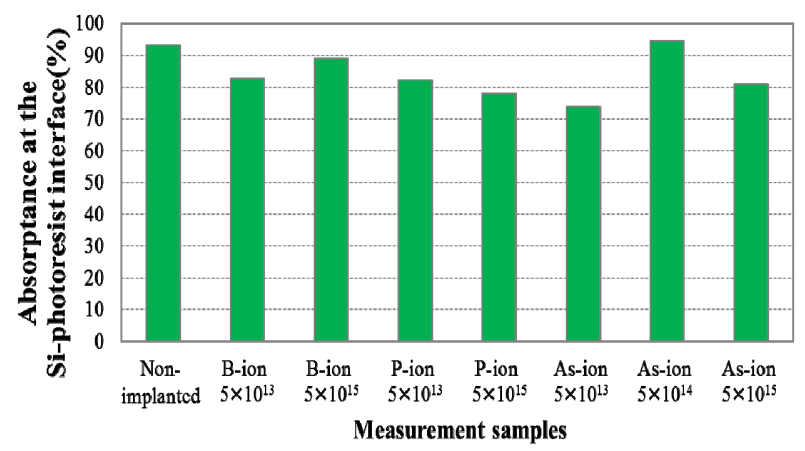

Figure 2. Optical characteristic of ion-implanted resist samples at $532 \mathrm{~nm}$ wavelength.

Resist stripping threshold (the point at which the resist begins stripping from the Si wafer) and laser-induced damage threshold (LIDT) of the $\mathrm{Si}$ wafer surface under the ion-implanted resist were measured at the laser wavelength of $532 \mathrm{~nm}$. In order not to generate laser damage to the Si wafer in this advanced laser resist stripping method, resist stripping threshold must be lower than the LIDT of the Si wafer surface coated with the ion-implanted resist. If the difference of these two thresholds is large, resist stripping is enabled in a safe laser irradiation condition. Table 1 shows the resist stripping threshold and LIDT of the Si wafer surface coated with the ion-implanted resist.

For the laser irradiation of 1 pulse, ion-implanted resist with a density of $5.0 \times 10^{13}$ atoms $/ \mathrm{cm}^{2}$ was completely stripped in the same way as that of non-implanted photoresist. The difference of the type of an implanted ion was not found in the resist stripping threshold obtained.

The resist stripping threshold was $42 \sim 48 \mathrm{~mJ}$. It was half a degree of the LIDT of the $\mathrm{Si}$ wafer surface coated with the ion-implanted resist. In case of the ion-implanted resist with a density of $5.0 \times 10^{15}$ atoms $/ \mathrm{cm}^{2}$, the resist stripping threshold decreased in 15 19 mJ. Only an outer side of the resist was stripped with 1 pulse irradiation. It is thought that optical absorption of the surface of the resist increased with a high-density ion implantation. The irradiated laser comes to be absorbed at the resist surface with the increase of the optical absorption of the resist. Therefore, after irradiating multiple pulses, the ion-implanted resist with a density of $5.0 \times 10^{15}$ atoms $/ \mathrm{cm}^{2}$ could be stripped without damaging the Si wafer.

Table 1. Optical characteristic of the resist sample in the second harmonics $(532 \mathrm{~nm})$ of a Nd:YAG laser.

\begin{tabular}{ccc}
\hline Sample name & $\begin{array}{c}\text { Photoresist stripping } \\
\text { threshold }\left(\mathrm{m} J / \mathrm{cm}^{2}\right)\end{array}$ & $\begin{array}{c}\text { LIDT at the Si-photoresist } \\
\text { intertace }\left(\mathrm{mJ} / \mathrm{cm}^{2}\right)\end{array}$ \\
\hline B-ion $5 \times 10^{13}$ & 42 & 98 \\
B-ion $5 \times 10^{15}$ & 12 & 65 \\
\hline$P$ ion $5 \times 10^{13}$ & 48 & 110 \\
\hline P-inn $5 \times 10^{15}$ & 15 & 41 \\
\hline As-ion $5 \times 10^{13}$ & 44 & 99 \\
As-ion $5 \times 10^{14}$ & 16 & 51 \\
As-ion $5 \times 10^{15}$ & 15 & 37 \\
\hline
\end{tabular}

Figure 3 shows a photograph of the processed surfaces when a laser beam with $532 \mathrm{~nm}$ was irradiated to the B ion-implanted resist in water. The energy of irradiated laser beam was adjusted to the intensity at which laser damage did not occur to the $\mathrm{Si}$ wafer surface. On the optimized condition, the non-implanted novolak resist was completely stripped by the laser irradiation with 1 pulse (Fig 3. (a)). The Boron ion-implanted resist 
with a density of $5.0 \times 10^{13}$ atoms $/ \mathrm{cm}^{2}$ was stripped similar way (Fig 3. (b)). The Boron ion-implanted resist with a density of $5.0 \times 10^{15}$ atoms $/ \mathrm{cm}^{2}$ was stripped by 20 pulses irradiation without occurring surface laser-induced damage (Fig 3. (c)). Therefore, it was found that even the highly ion-implanted resist could be stripped by using an advanced laser stripping method.

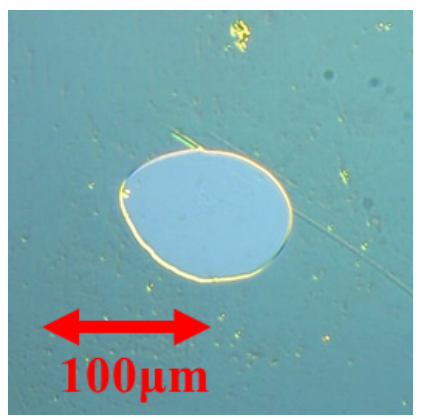

(a) non-implanted resist (1 pulse).

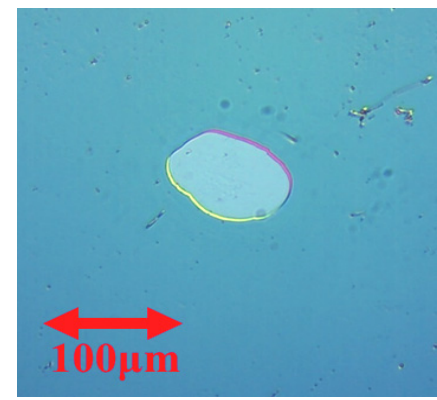

(b) Boron ion-implanted resist with density of $5.0 \times 10^{13}$ atoms $/ \mathrm{cm}^{2}$ ( 1 pulse).

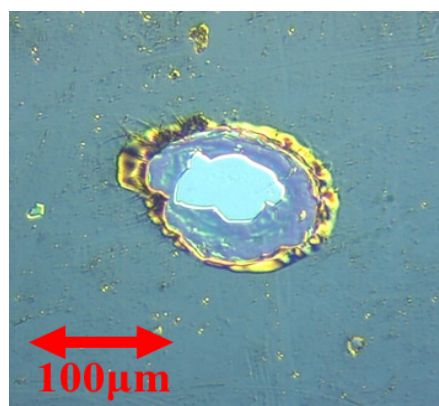

(c) Boron ion-implanted resist with density of $5.0 \times 10^{15}$ atoms $/ \mathrm{cm}^{2}$ (20 pulses).

Figure 3. An optical microscope photograph of the resist surface after irradiating with $532 \mathrm{~nm}$.

In addition, the ion-implanted resist stripping with laser beam scanning was carried out in the water. A pulsed $532 \mathrm{~nm}$ laser was irradiated to the boron ion-implanted resist (density of $5.0 \times 10^{15}$ atoms $/ \mathrm{cm}^{2}$ ) with 20 pulses per spot. The laser beams were scanned every $20 \mu \mathrm{m}$ after the laser irradiation of 20 pulses. The micro photography of the stripped ion-implanted resist surface after 532 $\mathrm{nm}$ laser scanning is shown in Figure 4. At the laser irradiated surface, the resist has been continuously stripped from the $\mathrm{Si}$ surface without occurring laser damage.

In contrast, the ion-implanted resist coated on the $\mathrm{Si}$ wafer remains on the surface without the laser irradiation. The stripping rate of the ion-implanted resist depends on the number of laser pulses. Thus, a laser light source with a large beam diameter and high repetition rate is required for its industrial application.

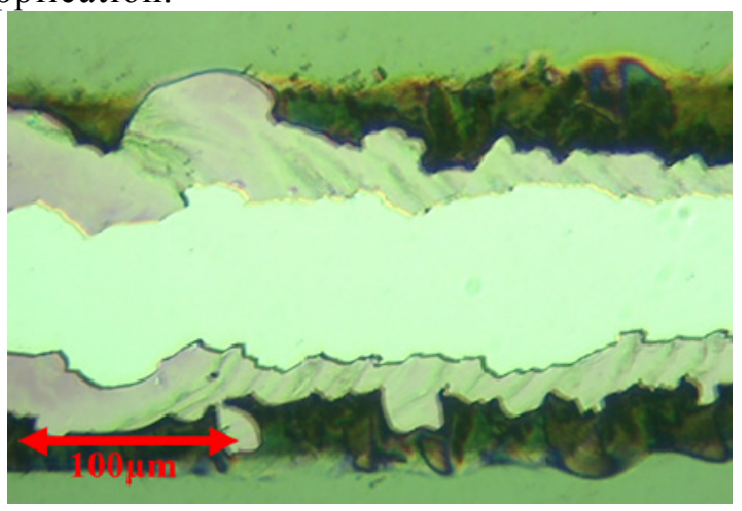

Figure 4. Micro photography for Boron ion-implanted resist with density of $5.0 \times 10^{15}$ atoms $/ \mathrm{cm}^{2}$ after $532 \mathrm{~nm}$ laser scanning.

\section{Conclusion}

We have investigated the ion-implanted photoresist removal by using an advanced laser resist stripping method. The optical characteristics of the ion-implanted resist were evaluated at the wavelength of $532 \mathrm{~nm}$. B, P, and As ion-implanted novolak resists with a density from $5.0 \times 10^{13}$ atoms $/ \mathrm{cm}^{2}$ to $5.0 \times 10^{15}$ atoms $/ \mathrm{cm}^{2}$ were prepared in this experiment. Regardless of implanted ion species and density, more than $74 \%$ of laser power was found to absorb into the $\mathrm{Si}$ wafer surface. A highly ion-implanted resist with a density of $5.0 \times 10^{15}$ atoms $/ \mathrm{cm}^{2}$ showed the absorbance of approximately $90 \%$ as the non-implanted resist. The difference of slight film thickness of the samples is thought to cause the dispersion of absorbance. For the laser irradiation of 1 pulse, the ion-implanted resist with a density of $5.0 \times 10^{13}$ atoms $/ \mathrm{cm}^{2}$ was completely stripped in the same way as 
that of non-implanted photoresist. In contrast, the ion-implanted resist with a density of $5.0 \times 10^{15}$ atoms $/ \mathrm{cm}^{2}$ was could be stripped by irradiating 20 pulses without damaging the $\mathrm{Si}$ wafer.

Furthermore, the ion-implanted resist stripping with laser beam scanning was carried out on B the ion-implanted resist with a density of $5.0 \times 10^{15}$ atoms $/ \mathrm{cm}^{2}$. The ion-implanted resist was continuously stripped from the Si surface without causing laser damage. From these results, the irradiation of the $532 \mathrm{~nm}$ pulsed laser to the resist in the water also enables ion-implanted resist stripping from the $\mathrm{Si}$ wafer. In addition, it is indicated that an advanced laser resist stripping method is a promising technique for reduction of environmental load in lithography process.

\section{References}

1. T. Miura, M. Kekura, H. Horibe, M. Yamamoto, and H. Umemoto, ECS Trans.19 (2009), 423.

2. K. K. Christenson, ECS Trans. 11 (2007), 197.

3. S. Fujimura, J. Konno, K. Hikazutani, and
H. Yano, Jpn. J. Appl. Phys. 28 (1989), 2130.

4. M. Yamamoto, T. Maruoka, A. Kono, H. Horibe, and H. Umemoto, Jpn. J. Appl. Phys. 49 (2010), 016701.

5. M. Yamamoto, T. Maruoka, Y. Goto, A. Kono, H. Horibe, M. Sakamoto, E. Kusano, H. Seki, and S. Tagawa., J. Electrochem. Soc., 157(3) (2010), H361-H370.

6. Huynh, C.K,; Mitchener, J. C., J. Vac. Sci. Technol, B9(2) (1991), 353-356.

7. Gardner,W.L., Baddorf,A.P., and Holber, W.M., J. Vac. Sci. Technol., A15(3)(1997), 1409- 1412.

8. Y. Goto, Y. Angata, M. Yamamoto, T. Seki, J. Matsuo, and H. Horibe, J. Photopolym. Sci. Technol., 26(4) (2013) $467-472$.

9. H. Horibe, M. Fujita, I. Nishiyama, and A. Yoshikado, J. Appl. Phys., 44(12), (2005), 8673-8675.

10. H. Horibe, T. Kamimura, T. Hata, M. Yamamoto, I. Yamato, O. Nigo, M. Fujita, A. Yoshikado, and K. Yoshida, Polymer J., 37(11), (2005), 813-817.

11. T. Kamimura, H. Muraoka, Y. Matsura, and H. Horibe., J. Photopolym. Sci. Technol., 25(6) (2012) 741-746. 